\title{
Understanding Task-Technology Fit in Smartphone Appointment Booking System Adopted by Chinese Hospitals Ci-Yong WANG ${ }^{1, a,{ }^{*}}$ and An-Ding $\mathrm{ZHU}^{2}$
}

\author{
${ }^{1}$ Information Centre, Zhejiang Cancer Hospital, Hangzhou 310022, China \\ ${ }^{2}$ Sch. of Mgmt. \& E-Bus., Zhejiang Gongshang University, Hangzhou 310018, China \\ awangcy@zjcc.org.cn \\ ${ }^{*}$ Corresponding author
}

Keywords: Task-Technology Fit, Crowd Queue Management, Smartphone Appointment Booking System.

\begin{abstract}
Smartphone is improving the quality of public services in China. Queuing of hospitals is a big problem in the past. But now, with the population of smartphone, patients can easily log in the remote booking servers anywhere and anytime. This study applies the Task-Technology Fit (TTF) theory to reveal what is major factors lead to users' experience. With the questionnaire and Structural Equation Modeling (SEM) approach, this study finds that multisource queuing, anti-cheating queuing, ubiquitous access, and universal interface are the major factors significantly impact on the TTF perception. And TTF perception is the predictor to the users' booking performance. This study will give implications for the third-party appointment and booking companies to develop such a kind of hospital information system.
\end{abstract}

\section{Introduction}

China has a large population. With the rapid urbanization in the last decade, megacities come into being. People find more opportunities in the city. Their children are offered better education as well. The concentration of population also makes social problems. The traffic block, the air pollution, and the roaring real-estate price go along with the big difficulty of hospitalization [1]. Patients find trouble in getting the opportunity to see the doctor. Immoral brokers see the chance to make money by controlling the registering system [2]. As the smartphone becomes more and more popular, the smartphone appointment booking system (SABS) help hospitals to fight against the immoral brokers.

SABS is one kind of the third party information systems that serve the hospitals, because patients need a uniform interface to operate, while the hospitals need to cut down the operation cost of taking customers in fairly. Various approaches have been tried before the emergency of smartphone [3]. Physically queuing in front of the acceptance window of the hospital is the classical approach to arrange patients in order and has been lasting for several decades. Plenty of time is wasted in queuing and people always dispute the fairness [4]. In order to seize a prior position, people need to line up very earlier in the morning. This situation brings into birth the immoral service for professional queuing. Later, telebooking systems [5] and online booking systems [6] once emerged to solve the queuing problem. However, they are not good solutions and died off due to the high maintaining cost.

On the side of patients and their kin, the efficiency, convenience, as well as fairness are the most important factors impacting their satisfaction and endurance [7]. Nowadays, every person has a smartphone in hand. Its functions include not only the vocal communication but also the apps downloaded from the app store to fulfill various kinds of tasks. Moreover, the smartphone sticks to the user physically. It carries all sources of information, attributions, and identities of the user and records his trajectories. It also gives the user a chance to response directly and quickly to the updated states from the apps [8].

However, why do the hospitals adopt the SABS technology to fulfill their purposes on appointing patients? And how do they reach these ends? These questions still remain unknown. In this study, we 
suppose to reveal the antecedents of the adoption based on the task-technology fit theory. In the next sections, we will construct the model and setup the hypotheses and test them by data collected from questionnaires. Conclusions and discussion will be conducted at the final section of the paper.

\section{The model and hypotheses}

The Task-Technology Fit (TTF) model is used for the purpose of this study. TTF was the contribution of Goodhue on the information system realm [9]. Goodhue argues that an information technology is adopted because of the good fit with the task rather than only utilization [10]. In fact, desktop computers can also be utilized for hospital booking and appointment. But, smartphones are better fit with the booking.

TTF is the classical framework to identify the influence factors of hospital adoption of variety of information systems. Lepanto et al. [11] compare the different performance perception between developers and radiologists toward a picture archiving and communication system (PACS) upgrade. The results show that TTF predicts utilization and perceived net benefits and TTF is a valid tool to assess perceived benefits, but it is important to take into account the characteristics of users. Chen et al. [12] used TTF model to access the "match" between the information-sharing tasks and HIS, RIS, PACS systems. They found that hospital information systems could support the CT patient-referral mechanism, increase hospital performance, reduce patient waiting time, and enhance the quality of care for patients. Gloria and Sueanne [13] identified four constructs of task characteristics, technology characteristics, individual use behavior, and environmental characteristics to frame the medication errors reduction. Chang et al [14] applied the TTF theory, system satisfaction, and postacceptance continuance models to reveal the users' assessment of the long-term care information system primarily depended on the system quality, locatability of data, timeliness, ease of use, and system-user relationship.

In addition, Merschbrock et al. [15] reported that the building information modeling technology combined with game development is related to the collaboration among construction, operational and gaming experts, clear communication of information needs, and better contractual agreements in the scenario of healthcare building education. Handayani et al. [16] adopted TTF model to explore the relationship among institutional isomorphism, institutional logic, and institutional entrepreneurship. They found that three factors of self-efficacy, social influence, and management support have a significant influence on the individual acceptance of hospital information system. Zakaria and Yusof [17] implemented a case study approach by using an in-depth interview with multidisciplinary medical team members to reveal that the less redundancy, data retrieval and storage have the positive relation to the new hospital information system and healthcare workers show positive attitudes during training and throughout the learning process. Ali [18] explored the portal help the patient users to complete health information management tasks, perceive usable, and succeed in setting up proxy accounts, which give them a clear understanding of tasks that they could accomplish. Ahmad et al. [19] argued that in Malaysia, hospitals adopt HIS system because of the factors of relative advantage, compatibility, security concern, hospital size, mimetic pressure-competitors, vendor support, perceived technical competence of IS staff, and employee's IS knowledge. Handayani et al. [20] focused on the Indonesia hospitals accept HIS systems considering human, technological, and organizational characteristics for supporting government eHealth programs. They found that human characteristics (i.e. compatibility, information security expectance, and self-efficacy), and organizational characteristics (i.e. management support, facilitating conditions, and user involvement) significantly influenced users' opinions of both the ease of use and the benefits of the HIS.

As shown in Fig. 1, we suppose that the task of booking or registering for hospital services includes three characteristics: multisource queuing, anti-cheating queuing, and instant synchronization. Multisource queuing refers that patients need not go to the acceptance windows of the hospitals physically. They are allowed to book via at least four channels: on-site, webpage, smartphone app, and telephone. Every channel has its own technical features of interface, delay, storage, and 
data-exchange. Since patients are diverse and different channels are designed for different needs, the priority mechanism is very important to be applied to queue these requests. Anti-cheating queuing refers that the booking system needs to prevent the illegal brokers and snatching apps from lining in the priority positions. If these agents find the crack they will destroy the fairness of the booking system and patients having the real needs turn to them with the high price. Instant synchronization means that the system needs to keep all the booking channels under the same pace. Sometimes, the system must balance all the channels and reserve some vacancies for IT technical illiterates.

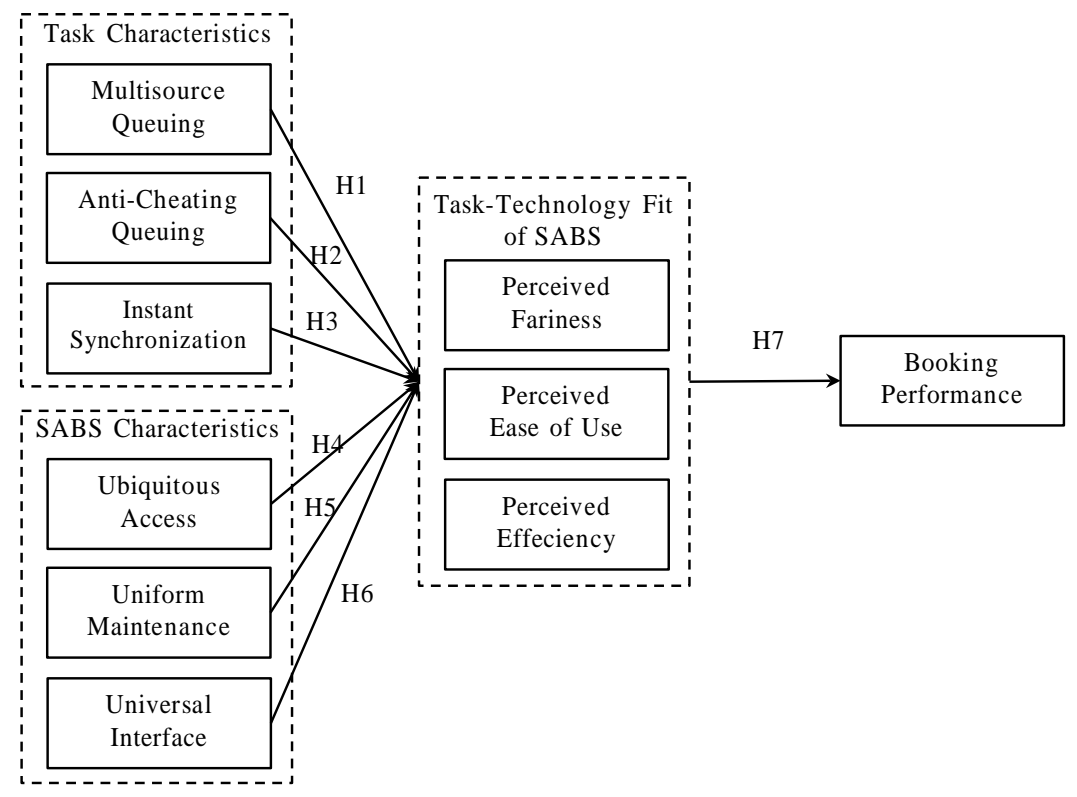

Fig. 1 The SABS model based on TTF model

We identify three kinds of SABS characteristics: ubiquitous access, uniform maintenance, and universal interface. Ubiquitous access refers that users can log into the system anywhere there is Internet connection. Smartphone is the proper personal device to connect to Internet. Uniform maintenance guarantees the quality and stabilization of the SABS system by a general third-party company. Universal interface means that patients have the same operation interface and easily learn how to use it.

To the users, TTF means it easy to use the SABS system. They need not study hard and then switch to the new system. They feel it is efficient after they use the SABS system to fulfill their boring booking requirements. And they perceive fairness as they know that the system cannot treat them. Thus, finally, they assert the SABS system can improve their booking performance.

\section{Data Collection}

Table 1. Results of hypotheses testing.

\begin{tabular}{|c|c|c|c|c|}
\hline \multirow{2}{*}{ Hypothesis } & \multirow{2}{*}{ Parameter } & \multicolumn{3}{|c|}{ Results of Hypothesis Testing } \\
\cline { 3 - 5 } & & Estimate & $p$ & Result \\
\hline H1 & TTF $\leftarrow$ MQ & 0.791 & 0.002 & Accepted \\
\hline H2 & TTF $\leftarrow$ ACQ & 0.080 & 0.004 & Accepted \\
\hline H3 & TTF $\leftarrow$ IS & 0.222 & 0.269 & Rejected \\
\hline H4 & TTF $\leftarrow$ UA & 0.103 & 0.011 & Accepted \\
\hline H5 & TTF $\leftarrow$ UM & 0.492 & 0.526 & Rejected \\
\hline H6 & TTF $\leftarrow$ UI & 0.372 & 0.049 & Accepted \\
\hline H7 & BP $\leftarrow$ TTF & 0.770 & 0.003 & Accepted \\
\hline
\end{tabular}


This study uses a questionnaire as the research instrument (Appendix A) with 7-point Likert scale. The respondents handed in 305 questionnaires with 16 uncompleted. The data was processed using Structural Equation Modeling (SEM). The results of hypotheses testing are listed in Table 1.

\section{Conclusions and discussion}

Multisource queuing is verified as significantly affecting the TTF perception. This result means that most respondents agree SABS systems give variety choices for users to access the system. Anti-cheating queuing has significant effect on the TTF perception, too. This result means that most respondents need the SABS system be trustworthy if it prevent cheating. The relationship between instant synchronization and TTF is not significant yet. It may be caused as most respondents do not know the background mechanism of SABS systems. Ubiquitous access has significant influence on TTF perception. Most respondents agree that they can retrieval information and status everywhere and every time as they need. Uniform maintenance does not significantly impact on TTF perception. This means that most users may not care about the operation and maintenance of the third-party service provider. Universal interface has significant effect on TTF perception. That is, most respondents think that smartphone can provide the easy way to operate the booking app and is a very suitable device for users to fulfill their needs of registering. At last, the TTF discriminant can predict users’ perception of booking performance. They are satisfied with the new SABS system.

\section{Appendix}

\section{Multisource Queuing (MQ)}

1. I know there are several channels to log in the booking system.

2. I know all the channels are under the same queuing mechanism.

3. I know there are other users compete with me in the other channels.

\section{Anti-Cheating Queuing (ACQ)}

1. I know the system has technology to check the illegal brokers out.

2. I know the system can tell the normal requests from the cheating ones.

3. I know the system can protect our real demand.

\section{Instant Synchronization (IS)}

1. I know the booking operation will be instantly pushed into the queue.

2. I know the booking operation will be merged with other channels.

3. I know the system has a reliable mechanism to synchronize all the channels.

\section{Ubiquitous Access (UA)}

1. I know that I can use smartphone to book everywhere.

2. I know that I can use smartphone to book every time.

3. I know that I can use smartphone to book via any Internet connection.

\section{Uniform Maintenance (UM)}

1. I know that the system is running by a general company.

2. I know that the company provides service for several hospitals.

3. I know that the company is a third-party company that needs one maintenance team.

\section{Universal Interface (UI)}

1. I know that the system has a universal interface.

2. I know that the system can be used by following the similar operation steps.

3. I know that the system has a series of similar settings.

\section{Task-Technology Fit (TTF)}

1. I perceive the system provide me a fair opportunity to book.

2. I perceive it is easy to use the booking system.

3. I perceive using the system is efficient.

\section{Booking Performance (BP)}

1. I think the booking system is of high performance. 
2. I am satisfied with the booking system.

3. I think the booking system is very useful.

\section{References}

[1] X. P. Zhu, R. Miao, and Z. B. Jiang. Research \& implementation of outpatient appointment and queuing management system in hospital based on lean thinking, Industrial Engineering \& Management, 12(6) (2008) 108-113.

[2] http://sh.qq.com/a/20170916/006536.htm (News in Chinese language)

[3] J. Q. Chi. Development and practice of hospital queuing system. China Medical Devices, 1 (2013) 52-54.

[4] N. Li, N. Kong, Q. Li, and Z. Jiang. Evaluation of reverse referral partnership in a tiered hospital system - A queuing-based approach. International Journal of Production Research, 1 (2017) 1-17.

[5] X. D. Ge, J. T. Lu, S. H. Feng. Design and implementation of calling and queuing system for outpatient triage. Chinese Medical Equipment Journal, 11 (2012) 46-49.

[6] X. J. Jiang, H. Zhou. Design and discussion of the online hospital registration booking system. Chinese Journal of Medical Device, 12 (2005) 17-19.

[7] D. Ou, F. Zeng, F. Liu, and X. B. Luo. Suggestions for employment of hospital queuing system. Chinese Medical Equipment Journal, 9 (2010) 104-105.

[8] O. L. Franko, T. F. Tirrell. Smartphone app use among medical providers in ACGME training programs. Journal of Medical Systems, 36(5) (2012) 3135-3139.

[9] D. L. Goodhue. Understanding user evaluations of information systems. Management Science 41(12) (1995) 1827-1844.

[10] D. L. Goodhue, R. L. Thompson. Task-technology fit and individual performance. MIS Quarterly, 19(2) (1995) 213-236.

[11] L. Lepanto, C. Sicotte, P. Lehoux. Assessing task-technology fit in a PACS update: Do users' and developers’ appraisals converge? Journal of Digit Imaging, 24 (2011) 951-958.

[12] P. S. Chen, C. J. Yu, G. Y. H. Chen. Applying task-technology fit model to the healthcare sector: A case study of hospitals' computed tomography patient-referral mechanism. Journal of Medical System, 39 (2015) 80.

[13] P. W. Gloria, M. Sueanne. Beyond technology adoption: An embeddedness approach to reduce medication errors. 25(2) (2015) 213-232.

[14] I. C. Chang, C. H. Chang, J. W. Wu, T. C. K. Huang. Assessing the performance of long-term care information systems and the continued use intention of users. Telematics and Informatics, 32 (2015) 273-281.

[15] C. Merschbrock, A. K. Lassen, T. Tollness, B. E. Munkvold. Serious games as a virtual training ground for relocation to a new healthcare facility. Facilities, 34 (13/14) (2016) 788-808.

[16] P. W. Handyani, A. N. Hidayanto, D. Ayuningtyas, I. Budi. Hospital information system institutionalization processes in Indonesian public, government-owned and privately owned hospital. International Journal of Medical Informatics, 95 (2016) 17-34.

[17] N. Zakaria, S. A. M. Yusof. Understanding technology and people issues in hospital information system (HIS) adoption: Case study of a tertiary hospital in Malaysia. Journal of Infection and Public Health, 9 (2016) 774-780. 
[18] S. B. Ali, J. Romero, K. Morrison, B. Hafeez, J. S. Ancker. Focus section health IT usability: Applying a task-technology fit model to adapt an electronic patient portal for patient work. Applied Clinical informatics, 9(1) (2018) 174-184.

[19] H. Ahmadi, M. Nilashi, L. Shahmoradi, O. Ibrahim. Hospital information system adoption: Expert perspectives on an adoption framework for Malaysian public hospitals. Computers in Human Behavior, 67 (2017) 161-189.

[20] P. W. Handayani, A. N. Hidayanto, A. A. Pinem, I. C. Hapsari, P. I. Sandhyaduhita, I. Budi. Acceptance model of a hospital information system. International Journal of Medical Informatics, 99 (2017) 11-28. 